\title{
"LA LUCHA DEL ESTADO PERUANO CONTRA EL TRABAJO INFANTIL Y LA TRATA DE NIÑOS, NIÑAS Y ADOLESCENTES: AVANCES Y DESAFÍOS"
}

\author{
VEGA LUNA, Eduardo ${ }^{304}$
}

SUMARIO: 1. Datos sobre la problemática de la niñez y el trabajo infantil. - 2. Responsabilidad del Estado frente al trabajo infantil. - 3. Recomendaciones específicas a los Estados para la protección de la niñez contra el trabajo infantil. - 4. Acciones complementarias por el Estado Peruano. - 5. El grave problema de la trata de niños y niñas con fines de explotación laboral. -6 . Reflexiones finales.

\section{RESUMEN}

Hacia 2011 la población infantil y adolescente era del 36\% concentrando los índices más altos de pobreza y los que trabajaban era de un millón setecientos noventicinco mil, de los cuales el 33.9\% realizaban trabajos de riesgo con más de 36 horas semanales y pese a las iniciativas del Estado peruano aún queda mucho por hacer.

Palabras clave: Trabajo infantil, trata de menores, explotación laboral, trabajo forzado.

\begin{abstract}
By 2011 the child population was $36 \%$ concentrating the highest rates of poverty and working was one million seven hundred ninety five thousand, of which $33.9 \%$ risk undertaking work over 36 hours a week and despite the Peruvian state initiatives is still much to do
\end{abstract}

Key words: Child labor, child trafficking, labor exploitation, forced labor.

304 Abogado por la Universidad Nacional Mayor de San Marcos. Estudios de Maestría en Paz y Desarrollo en la Cátedra UNESCO de filosofía para la Paz de la Universidad de Jaume I de España y estudios de Maestría en Derecho Penal por la Universidad Nacional Mayor de San Marcos. Defensor del Pueblo (e). 


\section{Algunos datos sobre la problemática de la niñez y el trabajo infantil}

De acuerdo a información de UNICEF correspondiente al año 2011, la población del Perú se acercaba a los 30 millones de personas. De este total, el $36 \%$ eran niños, niñas y adolescentes, es decir 10 millones 571 mil 879 personas. ${ }^{305}$

Además, los niños, niñas y adolescentes son los que concentran los índices más altos de pobreza. Mientras un 35\% del total de la población peruana vive en situación de pobreza, esta cifra se eleva a $49 \%$ entre los niños y niñas de 0 a 5 años, a $48 \%$ entre los de 6 a 11 años y a $41 \%$ entre los de 12 y 17 años. En total, alrededor del $45 \%$ del total de niños, niñas $\mathrm{y}$ adolescentes en nuestro país viven en condiciones de pobreza. ${ }^{306}$

Por otro lado, según los resultados de la última Encuesta Nacional de Hogares (ENAHO) elaborada por el Instituto Nacional de Estadística e Informática - INEI, la población de niñas, niños y adolescentes que trabajaba en nuestro país el año 2011 era de 1 millón 795 mil; es decir, un 4,3\% menos que la que existía el año 2007.

De esta cifra, 832, 000 niños y niñas trabajadores tienen entre 6 y 13 años de edad; mientras que 826, 000 tienen entre 14 y 17 años. El 33,9\% de adolescentes en el país realiza trabajos de riesgo y le dedica más de 36 horas semanales a dichos oficios. Ello, sin tomar en cuenta la "cifra negra" de los casos no registrados.

En ese sentido, si bien se reconocen las iniciativas adoptadas por el Estado peruano en la protección de los derechos de la niñez y adolescencia; aún queda mucho trabajo por hacer, ya que la cifra de trabajo infantil y adolescente todavía es elevada.

Para graficar este grave problema, me permito narrarles dos situaciones, entre las muchas que llegan a conocimiento de nuestra institución.

En la primera de ellas, dos adolescentes de 13 y 15 años viajaron de Andahuaylas a Abancay (región Apurímac), con el permiso de sus padres, para buscar trabajo. En dicha ciudad, se encontraron con un hombre que les ofreció trabajar en su panadería. Luego de dos semanas, el adolescente de 13 años sufrió un grave accidente en su mano izquierda al manipular una de las máquinas para amasar pan y, debido a la gravedad de la lesión, terminaron por amputarle dos dedos. Las autoridades advirtieron que este adolescente era explotado aproximadamente 14 horas al día, especialmente por las noches. Conocidos estos hechos, se realizó un operativo en la referida panadería, en el que se logró rescatar al otro adolescente.

En el segundo caso, dos adolescentes -de 14 y 17 años de edad- se presentaron en la sede del Ministerio Público en Huacho para denunciar a una pareja de esposos que las había convencido de salir de su ciudad natal, Juliaca en Puno, para trabajar vendiendo churros. Sin embargo, cuando llegaron a la casa de la pareja, en Huacho, empezaron a recibir maltratos físicos y psicológicos y no se les pagó nada de lo prometido, lo que las motivó a escapar. Posteriormente, las investigaciones policiales determinaron que las víctimas trabajaban 18 horas al día y que en esa casa existían más menores de edad explotadas.

\footnotetext{
${ }^{305}$ Información contenida en el documento de UNICEF: “Estado de la niñez en el Perú”. Lima, 2011.

${ }^{306}$ Información contenida en el documento de UNICEF: "Estado de la niñez en el Perû". Lima, 2011. p. 10.
} 
Estos casos ilustran la grave problemática del trabajo infantil en nuestro país. Situación que también ha sido reseñada en un informe del Departamento de Estado Norteamericano del 2012, que da cuenta que el trabajo infantil forzado en el Perú continúa existiendo, especialmente en la minería informal de extracción de oro, entre grupos de mendigos en las zonas urbanas, en el servicio doméstico y en la producción y el transporte de cocaína.

Lamentablemente, hechos como éstos son comunes en nuestra sociedad, hasta el punto de generar indiferencia. Niños, niñas y adolescentes de todas las edades realizan actividades incompatibles con su edad - que incluso pueden tener relevancia delictivadebido esencialmente a la situación de extrema pobreza que atraviesan sus familias.

\section{La responsabilidad del estado frente al trabajo infantil}

Frente a la problemática del trabajo y la explotación infantil, la actuación del Estado debe ser de un enérgico rechazo a situaciones que vulneren los derechos de nuestros niños, niñas y adolescentes; que -como todos sabemos- son titulares de derechos y obligaciones, que deben ser respetados por el Estado y la comunidad.

Esta nueva visión de la infancia, conocida como la "Doctrina de la Protección Integral", se desprende del texto de la Convención sobre los Derechos del Niño, ratificado por nuestro país el año 1990 y que -en la actualidad- es el instrumento internacional más importante sobre niñez. Antes de la Convención, los niños, niñas y adolescentes eran considerados como meros objetos de protección, a quienes se les debía asistir por razones de piedad, lo que se conocía como la "Doctrina de la Situación Irregular."

Es preciso incidir en estas definiciones. Y es que, a pesar que normativamente se ha establecido que los niños, niñas y adolescentes son sujetos de derecho, en la práctica, muchas veces las autoridades hacen caso omiso a la ley, desconociendo a los niños, niñas y adolescentes como personas capaces de ejercitar sus derechos y reclamar el cumplimiento de los mismos.

Esta situación es lo que Mary Beloff ${ }^{307}$ denomina un "fraude de etiquetas" en relación con la infancia y la adolescencia; es decir, situaciones en las que las modificaciones normativas sólo implican un cambio de nombre, vacío de contenido, y no un cambio en las prácticas y políticas institucionales.

Es a partir de esta nueva visión que se debe abordar la grave problemática del trabajo y la explotación infantil. Lamentablemente, este es un problema de larga data en nuestras sociedades que, incluso, ha sido objeto de análisis de la Organización Internacional del Trabajo desde sus inicios. Así, podemos mencionar que el Convenio $\mathrm{N}^{\circ} 5$ de la OIT, publicado en 1919, abordó el tema de la edad mínima de acceso al empleo en la industria.

Actualmente, existen dos instrumentos internacionales claves sobre la materia. El primero es el Convenio $\mathrm{N}^{\circ} 138$ de la OIT, referido a la edad mínima de acceso al empleo, en el que se fijan las edades mínimas requeridas según los tipos de trabajo. Este Convenio tiene como objetivo expreso "la total abolición del trabajo de los niños".

\footnotetext{
${ }^{307}$ Especialista argentina en materia de infancia y adolescencia.
} 
El segundo instrumento internacional de especial relevancia es el Convenio 182 de la OIT sobre las peores formas de trabajo infantil. Este instrumento exige que los Estados adopten medidas inmediatas y eficaces para conseguir la prohibición y eliminación de las peores formas de trabajo infantil, entendidas como aquellas actividades que atentan gravemente contra los derechos fundamentales de los niños, niñas y adolescentes. Entre éstas se encuentran la esclavitud, la trata de personas, la servidumbre por deudas, el trabajo forzoso u obligatorio, el reclutamiento o la oferta de niños para la prostitución, entre otros. Además, el Convenio 182 invoca a los Estados miembros a que implementen los respectivos mecanismos de vigilancia y programas de acción para erradicar estas prácticas.

Como se advierte, existe una tendencia a nivel internacional a proscribir el trabajo infantil, especialmente aquellos calificados como "peligrosos", así como las denominadas "peores formas de trabajo infantil".

En el ámbito nacional, el artículo 23 de la Constitución Política establece que el trabajo -en sus diversas modalidades- es objeto de atención prioritaria del Estado. Asimismo, este artículo establece la obligación estatal de proteger especialmente a la madre, al menor de edad y al impedido que trabajan. De lo anterior, se advierte claramente la obligación del Estado de proteger a las personas menores de edad en el marco de las políticas públicas del sector trabajo.

Por su parte, el Código de los Niños y Adolescentes regula el régimen legal para el Adolescente Trabajador. Al respecto, se debe destacar lo dispuesto por el artículo $49^{\circ}$ del Código, que señala que la protección al adolescente trabajador corresponde al Ministerio de la Mujer y Poblaciones Vulnerables en forma coordinada y complementaria con los Sectores Trabajo, Salud y Educación, así como con los Gobiernos Regionales y Municipales. Además, de acuerdo al artículo $51^{\circ}$ de dicho Código, la edad mínima para que los y las adolescentes accedan a un trabajo en nuestro país, de acuerdo al régimen general, es de 14 años.

Adicionalmente, el Poder Ejecutivo -mediante Decreto Supremo $N^{\circ}$ 003-2010MIMDES- aprobó la lista de trabajos peligrosos que, por la exigencia de las labores, pueden interferir o comprometer la integridad, la seguridad o la moral de los y las adolescentes. Entre éstos cabe destacar a la actividad minera, construcción civil, industria de hidrocarburos, fabricación artesanal de ladrillos y adobes, aquellos realizados en centros nocturnos, prostíbulos, en la vía pública, etc.

La utilización de menores de edad en estos trabajos se encuentra prohibida, y tiene sanción administrativa y penal.

En suma, de la normativa expuesta, se aprecia que -tanto a nivel internacional y nacional- únicamente se permite el trabajo de los y las adolescentes de acuerdo a las edades mínimas señaladas en la ley. Adicionalmente, se debe garantizar que no exista explotación económica, que su realización no importe riesgo o peligro, ni afecte su proceso educativo o sea nocivo para su salud o para su desarrollo. 


\section{Recomendaciones específicas a los estados para la protección de la niñez contra el trabajo infantil}

La problemática del trabajo infantil ha merecido el pronunciamiento de diversos organismos de Naciones Unidas. El Comité de los Derechos del Niño, en su Observación General $\mathrm{N}^{\circ} 4$, titulada "La salud y el desarrollo de los adolescentes en el contexto de la Convención sobre los Derechos del Niño”, instó a los Estados parte a proteger a los y las adolescentes contra toda forma de trabajo que pueda poner en peligro el ejercicio de sus derechos; especialmente prohibiendo toda forma de trabajo infantil y reglamentando el entorno laboral y las condiciones de trabajo.

Además, este organismo supranacional ha emitido recomendaciones a nuestro país sobre el tema de trabajo infantil. En ese sentido, en las Observaciones Finales de los años 1993, 2000 y 2006, encargó al Estado peruano la implementación de diversas medidas, entre las que cabe destacar la ratificación de los Convenios 138 y 182 de la OIT, así como la elevación de la edad mínima de admisión al empleo a los 15 años.

Cabe destacar que esta última recomendación ya se encuentra recogida en el proyecto de modificación del Código de los Niños y Adolescentes que se viene debatiendo actualmente en el Congreso.

Por su parte, debe mencionarse que la situación de la niña trabajadora ha merecido un especial pronunciamiento por parte de la Asamblea General de la ONU. Así, en marzo del presente año, se invocó a los Estados a que garanticen que las niñas trabajadoras tengan acceso en condiciones de igualdad a un trabajo decente y a un sueldo y una remuneración equitativos; que estén protegidas de la explotación económica, la discriminación, el acoso sexual, la violencia y el maltrato en el lugar de trabajo, que conozcan sus derechos y tengan acceso a la educación académica y no académica, a la capacitación práctica y a la formación profesional.

Al respecto, comparto la preocupación expresada por la situación de las niñas y adolescentes trabajadoras, toda vez que, lamentablemente, ellas se encuentran más expuestas a diversas formas de discriminación y violencia, y corren mayor riesgo de padecerlas.

\section{Acciones implementadas por el estado peruano}

En los últimos años, el Estado peruano ha dado pasos importantes en la lucha por la erradicación del trabajo infantil. Entre ellos, podemos destacar la regulación sobre las edades requeridas para trabajar en determinadas actividades; así como normas que establecen sanciones para los empleadores que permiten el trabajo de los y las adolescentes por debajo de dichas edades.

En materia de planes, se aprobó el Plan Nacional de Prevención y Erradicación del Trabajo Infantil 2005-2010, así como los Planes Nacionales de Acción por la Infancia y la Adolescencia 2002-2010 y 2012-2021, en los que se planteó como objetivo central la protección de los niños, niñas y adolescentes frente al trabajo infantil.

De otro lado, se realizaron diversas acciones como la creación del Comité Directivo Nacional para la Prevención y Erradicación del Trabajo Infantil - CPETI, así como de comités regionales en esta materia. Asimismo, se institucionalizó el 12 de junio como el "Día 
contra el Trabajo Infantil". Recientemente, el Ministerio de Trabajo ha adoptado la "Estrategia sectorial para la prevención y erradicación del trabajo infantil en nuestro país", que consta de 12 acciones enfocadas en la protección de la niñez y la infancia en el ámbito laboral. Entre éstas cabe destacar la inclusión de un componente sobre trabajo infantil en los planes de responsabilidad social de las empresas a nivel nacional; el compromiso de los beneficiarios de los programas sociales para que sus hijos dejen de trabajar; así como la incorporación de acciones de inspección en materia de trabajo infantil, en los planes de fiscalización de las direcciones regionales de trabajo.

\section{El grave problema de la trata de niños y niñas con fines de explotación laboral}

Finalmente, debo referirme a una problemática que, si bien guarda relación con el problema del trabajo infantil, constituye un fenómeno autónomo: me refiero a la trata de personas con fines de explotación laboral.

Hablar de trata de personas es hablar de esclavitud, pues este delito parte de concebir a la persona como un mero objeto de comercio - disponible al mejor postordesconociendo lo más preciado que tiene un ser humano, que es su dignidad. Consiste concretamente en trasladar a una persona de su lugar de origen, recurriendo muchas veces al engaño, para que sea vilmente explotada en otro lugar.

Al respecto, el Convenio 182 de la OIT establece la trata de personas como una de las peores formas de trabajo infantil. Asimismo, la trata constituye una grave afectación a los derechos fundamentales de los niños, niñas y adolescentes y un execrable delito, cuya pena puede llegar hasta los 35 años.

Debemos tomar conciencia que la trata de personas constituye un grave problema que afecta, de manera especial, a los más vulnerables en la sociedad, como son los niños, niñas y adolescentes. Y es que, de acuerdo a UNICEF, actualmente casi 2,5 millones de personas en el mundo están sometidas a trabajos forzosos como resultado de la trata. De esta cifra, entre el $22 \%$ y el $50 \%$ de las víctimas de trata son niños y niñas.

Sin embargo, es preocupante constatar que existen diversos problemas en la judicialización y sanción de los casos de trata. De acuerdo al informe del Departamento de Estado Norteamericano del 2012, estos problemas se encuentran referidos -principalmente- a la confusión del delito de trata con figuras similares, a la falta de un adecuado sistema de registro de casos, a la falta de recursos necesarios para que la Policía Nacional investigue este delito, a la poca cantidad de casos de trata con fines de explotación laboral registrados, entre otros.

Ante esta grave situación, desde el año 2007, la Defensoría del Pueblo viene asumiendo un rol en la lucha contra la trata de personas. En el ámbito de nuestras competencias, hemos conocido 65 casos a través de quejas presentadas por ciudadanos y ciudadanas, así como por actuaciones de oficio.

Además, formamos parte del Grupo Multisectorial contra la Trata de Personas, que es la instancia nacional donde se adoptan las políticas públicas contra dicho flagelo y participamos en los diversos grupos regionales que se han creado en todo el país. Adicionalmente, a la fecha, venimos elaborando un informe defensorial sobre el tema de trata 
de personas, que tiene como objetivo central evaluar las políticas públicas adoptadas por el Estado peruano en la lucha contra este flagelo.

Dos propuestas para combatir esta grave situación. La primera de ellas, es proponer que se aumente la pena en los delitos contra la libertad de trabajo previsto en el artículo $168^{\circ}$ del Código Penal. De este modo, nuestro país se adecuará a los estándares de protección previstos en el Convenio $\mathrm{N}^{\circ} 29$ de la OIT, sobre trabajo forzado.

En efecto, a la fecha, un acto de violencia o grave amenaza contra un niño, niña o adolescente para obligarlo a trabajar, se encuentra sancionado únicamente con una pena no mayor a 2 años; lo que, en la práctica, implica que el culpable nunca será encarcelado. Así, se genera un círculo vicioso en el que la afectación de los derechos de los niños, niñas y adolescentes se ve agravada por la ausencia de mecanismos efectivos de prevención y sanción.

De otro lado, se propone ratificar el Convenio $\mathrm{N}^{\circ} 189$ sobre las trabajadoras y los trabajadores domésticos. Sin duda, este instrumento internacional constituye un mecanismo necesario para la lucha contra la trata de personas -en especial de los niños, niñas y adolescentes- con fines de explotación laboral en el ámbito doméstico. Cabe señalar que el ámbito doméstico es considerado el sector económico en el que existe menos supervisión de las condiciones de trabajo y del cumplimiento de las obligaciones laborales por parte de los empleadores.

\section{Reflexiones finales}

Llegado a este punto, quisiera hacer una reflexión general. De acuerdo a los instrumentos internacionales citados, a las opiniones del Comité de los Derechos del Niño, y a la legislación nacional vigente, el trabajo de niños y niñas se encuentra prohibido, mientras que en el caso de los y las adolescentes solo se permite a partir de los 14 años de acuerdo al régimen general, y únicamente para ciertas actividades. Fuera de estos supuestos, la utilización de menores de edad para actividades remuneradas o no remuneradas será ilegal, y podrá devenir incluso en situaciones de trata con fines de explotación laboral, o de trabajo forzado.

No obstante, consideramos que, al margen de ello, el objetivo al cual debe aspirar toda sociedad que busca la inclusión social es que los niños, niñas y adolescentes no deberían realizar ningún tipo de trabajo, ya que ello no corresponde a su edad. Antes bien, deberían dedicarse a estudiar y jugar, que es lo que les toca vivir en esa etapa de la vida.

Somos conscientes que muchas veces la difícil situación por la cual atraviesan las familias lleva a que los pequeños de la casa salgan a trabajar, con la intención de apoyar a la economía del hogar. Sin embargo, está demostrado que la mejor forma en que los niños, niñas y adolescente colaboren con el hogar y con el país es estudiando.

Así, de acuerdo a un estudio de la OIT del año 2003, se determinó que los costos en los que incurrirían los gobiernos nacionales y los hogares en la erradicación del trabajo infantil serían superados ampliamente en sus beneficios. En ese sentido, la eliminación del trabajo infantil en el mundo podría generar beneficios por 5,1 billones de dólares, en especial en los países con economías en desarrollo o en transición como el Perú. 
Por otro lado, actualmente, la lucha por la erradicación del trabajo infantil se ve fortalecida por el compromiso asumido en la última Conferencia Internacional del Trabajo realizada en Ginebra en junio de este año.

Esta tarea no es imposible. Al respecto, debo señalarles que la Oficial de Programas de la Organización Internacional de Trabajo, María Olave, recientemente destacó que el Perú es el único país en el mundo que ha logrado revertir una situación de trabajo infantil en la minería artesanal, con un ejemplo de modelo exitoso y sostenible de negocio.

Ella se refería a la experiencia de la comunidad Nueva Santa Filomena, en Ayacucho, en donde la práctica minera de extracción de oro se formalizó gracias al trabajo articulado entre el Estado, la cooperación internacional y la sociedad civil. Actualmente, dicha comunidad exporta oro libre de trabajo infantil.

Experiencias como estas nos llenan de esperanza, para que, en un futuro no muy lejano, podamos ver a nuestros niños, niñas y adolescentes disfrutar plenamente de la vida libre de violencia, explotación y trabajo.

Recordando las palabras de Karl Menninger, padre de la psiquiatría norteamericana: "lo que se les de a los niños, los niños lo darán a la sociedad." Construyamos desde hoy la sociedad del mañana y, para ello, aunemos nuestros esfuerzos para la protección de los derechos de nuestra niñez.

\section{BIBLIOGRAFIA}

- Código de los niños y adolescentes

- Constitución política del Estado

- Convenio N5 OIT 1919

- Convenio $\mathrm{N}^{\circ} 138$ OIT Edad mínima de acceso al trabajo

- Convenio $\mathrm{N}^{\circ} 182$ OIT Peores formas de trabajo infantil

- Convención sobre los derechos del niño 1990

- D. S. 003-2020-MIMDES. Lista de trabajos peligrosos

- Documento UNICEF" Estado de la niñez en el Perú 2011" 